\title{
Pan computed tomography for blunt polytrauma: Are we doing too many?
}

\author{
G V Oosthuizen, FCS (SA); J L Bruce, FCS (SA); W Bekker, FCS (SA); N Shangase, FCS (SA); G L Laing, FCS (SA), PhD; \\ D L Clarke, FCS (SA), PhD
}

Pietermaritzburg Metropolitan Trauma Service, Department of Surgery, School of Clinical Medicine, College of Health Sciences, Nelson R Mandela School of Medicine, University of KwaZulu-Natal, Pietermaritzburg, South Africa

Corresponding author: G V Oosthuizen (george.oost@gmail.com)

Background. Pan computed tomography (CT) is widely used in the evaluation of patients with blunt polytrauma, but there is growing concern about the radiation risks imposed.

Objectives. To ascertain whether we were possibly overutilising pan CT in our trauma service, and whether we could safely cut down on scans without missing significant injuries.

Methods. We audited all pan scans performed in the Metropolitan Trauma Service, Pietermaritzburg, South Africa, during the 12-month period 1 January - 31 December 2012. An analysis was done to determine what injuries were identified and how these findings influenced our management. Results. Of the 140 pan scans, 108 (77.1\%) influenced management. These included the following components: 62 brain scans ( $44.3 \%), 16$ cervical spine scans (11.4\%), 50 chest scans (35.7\%) and 31 abdominal scans (22.1\%). The remaining 32 pan scans (22.9\%) did not influence management. However, it turned out that many of these 'clinically negative' scans were in fact clinically important, ruling out injury in patients in whom clinical assessment was regarded as unreliable: 3 patients (2.1\%) were hypoxic and had to be sedated, intubated and ventilated; 14 (10.0\%) had a Glasgow Coma Score (GCS) of $<15$; and $9(6.4 \%)$ had major distracting injuries. This left only 6 pan scans $(4.3 \%)$ that were not regarded as clinically helpful. Conclusion. In our setting, the majority of pan scans influence management. By ruling out significant injuries, clinically negative scans are valuable in patients who are obtunded, intubated and ventilated, or have major distracting injuries. In patients with a GCS of 15, not sedated and ventilated and with no major distracting injuries, clinical assessment and alternative imaging modalities may suffice.

S Afr Med J 2016;106(8):801-803. DOI:10.7196/SAMJ.2016.v106i8.10376

The advantages of a pan (head-to-pelvis) computed tomography (CT) scan in the blunt polytrauma setting are manifold, including rapid identification of multiple injuries, improved prioritisation of management, guided selection of non-operative management of solid organ injuries, and better selection of patients needing intensive care or those who can safely be admitted to the ward or discharged home. Pan CT in this setting has been shown to have a positive impact on

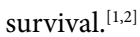

Unfortunately there are also a number of disadvantages to pan CT, including financial expense, potential overuse of limited resources, risks related to the use of intravenous contrast medium, and most worrisome, radiation risk. Relevant organ radiation doses from a fullbody CT examination range from 10 to $16 \mathrm{mGy}$ and result in a mean effective dose (weighted average over all relevant organs) of about $12 \mathrm{mSv}^{\left[{ }^{[3]}\right.}$ The estimated lifetime cancer mortality risk from a single full-body CT examination is $\sim 1: 1250$ for a 45 -year-old adult and $\sim$ 1:1 700 for a 65 -year-old adult. ${ }^{[3]}$ The estimated risk is considerably higher in a 1-year-old: $0.18 \%$ for abdominal CT and $0.07 \%$ for head CT. ${ }^{[4]}$ Although this may seem like a small risk to an individual, considering how many CT scans are being performed annually it constitutes a substantial population risk.
This poses the challenge that we need to be as selective as possible when using pan $\mathrm{CT}$, but without missing important injuries. To answer the question 'Can we safely cut down on pan scans?' we performed an audit of all the pan scans we performed in our metropolitan unit in 2012.

\section{Methods}

All data were retrieved from the prospectively maintained trauma registry in the Department of Surgery, University of KwaZulu-Natal, Pietermaritzburg, South Africa. Ethics approval was granted to perform retrospective audits from this registry (Biomedical Research Ethics Committee, No. 207/09). Our metropolitan trauma service comprises two busy urban hospitals (Edendale with 900 beds and Grey's with 500 beds; 2500 trauma admissions per annum combined). ${ }^{[5]}$ We performed an audit of all pan CT scans done for blunt polytrauma at both hospitals in 2012. Indications for pan CT in our setting include injuries on both sides of the diaphragm (e.g. head injury and fractured femur), significant mechanism of injury (e.g. fall from a height or ejected from a moving vehicle), and depressed level of consciousness with unknown mechanism of injury (Table 1). Only well-resuscitated, haemodynamically stable patients are allowed pan CT.

Table 1. Indication for pan CT in blunt trauma patients ${ }^{*}$

Injuries on both sides of the diaphragm (e.g. head injury and fractured femur)

Significant mechanism of injury (e.g. fell from a height or ejected from a moving vehicle) with evidence of polytrauma

Depressed level of consciousness with unknown mechanism of injury (e.g. found unconscious by the roadside)

*Patients must be haemodynamically stable. 


\section{Results}

A total of 140 pan scans were performed in our metropolitan unit during 2012. Of these, 108 were done on males and 32 on females. The average age of our patients was 24 years (range 2 - 62). The mechanism of injury was vehicle related in 119 patients, the remaining injuries being related to assaults, falls and building collapse.

Five pan scans (3.6\% of all pan scans performed) were totally negative, i.e. all components (head, neck, chest, abdomen, pelvis) were negative for any signs of injury. The remaining 135 scans (96.4\%) had positive findings of some sort, major or minor.

Of the 140 pan scans, 108 (77.1\%) demonstrated findings that influenced our management. These included the following components: 62 brain scans $(44.3 \%$ of the total), 16 cervical spine scans (11.4\%), 50 chest scans (35.7\%) and 31 abdominal scans (22.1\%) (Fig. 1).

The remaining 32 pan scans $(22.9 \%)$ were either 'radiologically negative' or demonstrated findings that did not influence management (Fig. 2). To facilitate further analysis, these scans are referred to as 'clinically negative. Among the patients who had clinically negative scans, 3 (2.1\%) were intubated and ventilated, admitted to the ICU, and kept sedated for $>24$ hours, due to hypoxia related to either lung contusion or aspiration or both. A further 14 patients $(10.0 \%)$ had a Glasgow Coma Score (GCS) of $<15$ and could not be reliably assessed clinically, while 9 patients (6.4\%) had major distracting injuries that made assessment of the cervical spine and abdomen unreliable. This left only 6 pan scans (4.3\%) that were not regarded as clinically helpful; these patients were not sedated and ventilated, did not have a GCS of $<15$, and did not have major distracting injuries.

\section{Discussion}

There is little doubt about the radiation risks associated with CT imaging, and in particular the use of pan CT. ${ }^{[6-10]}$ In a modern age where many imaging modalities involve radiation, the radiation burden to the population is already high. ${ }^{[1]}$ CT has become the imaging modality of choice for a wide variety of medical conditions, and unfortunately carries a considerably higher radiation burden than other imaging modalities that involve radiation. ${ }^{[12]}$

Furthermore, CT has become a leading imaging modality in emergency rooms, and patients may be exposed to repeated CT scanning at separate visits to the emergency room. ${ }^{[13]}$ Also of concern is the practice of repeat CT scanning of patients who have

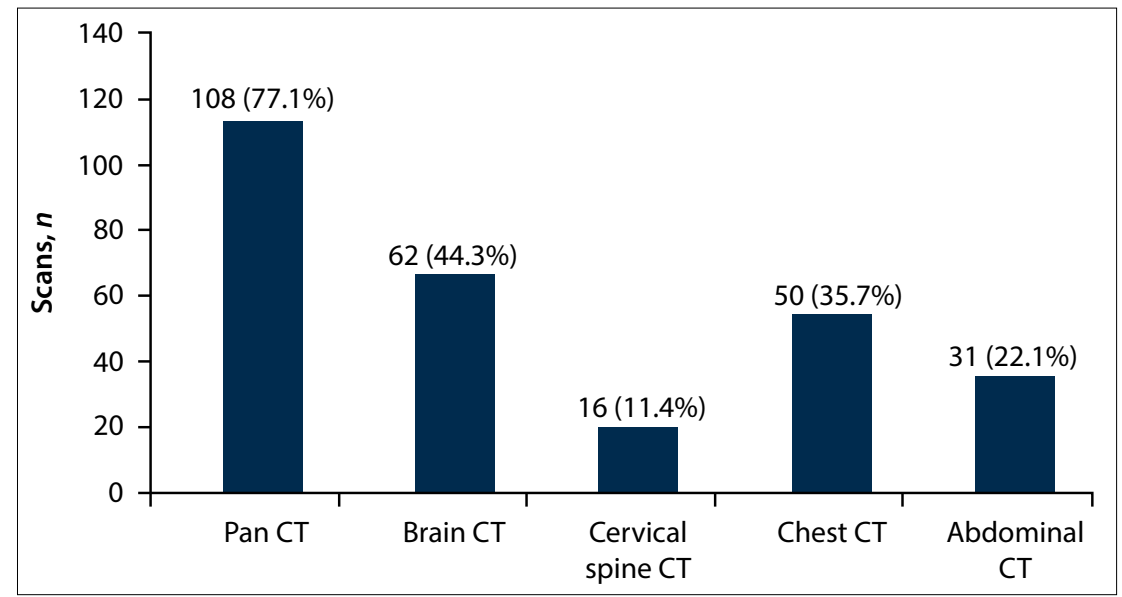

Fig. 1. Pan scans/components that influenced initial management.

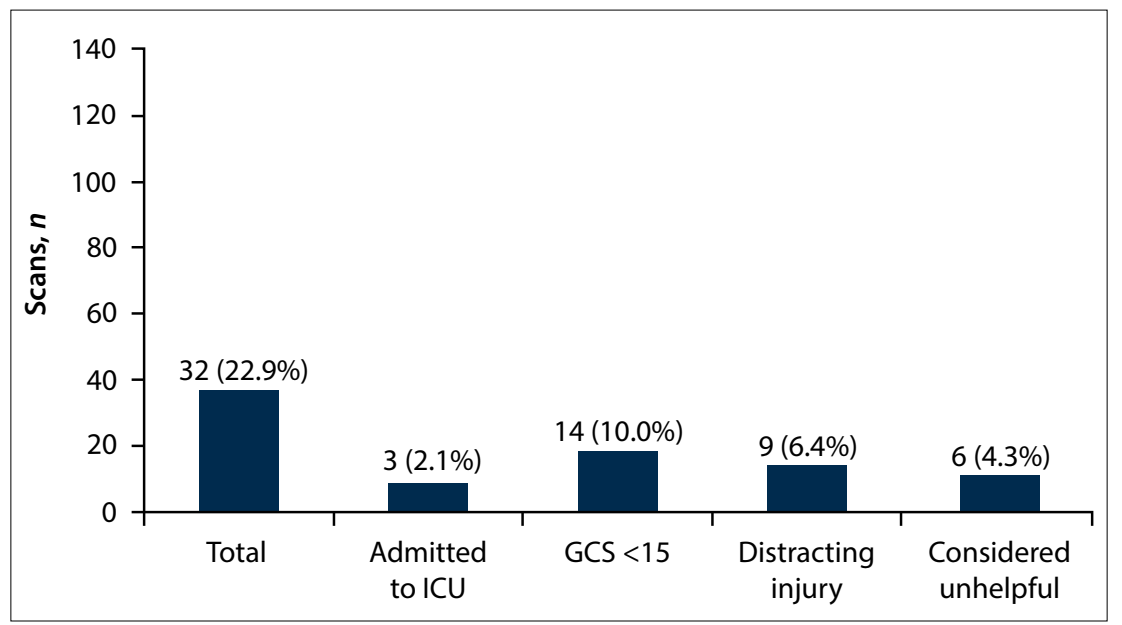

Fig. 2. Pan scans that did not influence initial management.

been referred from another centre where they have already been scanned but the images on the transferred storage disc are not compatible with the receiving centre's technology. ${ }^{[14]}$

Balanced against the above concerns, modern trauma care has become inseparable from pan CT; the concept is embraced worldwide and the benefits are clear. However, in light of the risks, in particular radiation risk, clinicians have to weigh the risk v. the benefits for each individual patient.

The overriding concern is that of possible overuse of this imaging modality. Salim et al. ${ }^{[15]}$ advocate a liberal policy in which pan CT is advocated for all patients with significant mechanisms of injury, even when they are clinically evaluable with no obvious signs of chest or abdominal injury. In their study, overall treatment was changed in $18.9 \%$ of patients based on abnormal CT scan findings. The concern arises that $>80 \%$ of their patients could have been spared a pan scan, and the question arises whether other modalities may equally guide management in this setting.
Imaging of polytrauma patients at our institution is undertaken according to strict protocols, in an attempt to ensure that unnecessary investigations are minimised and that inappropriate patients are not sent for imaging. In our analysis, over three-quarters (77.1\%) of scans influenced initial management of this cohort of trauma patients. This left $22.9 \%$ of patients in whom there were some CT findings that did not alter the initial management. While these scans were not necessarily radiologically negative, we considered them to be clinically negative.

The concern arises that these clinically negative scans may have been unnecessarily performed, and that we could have spared this group of patients the radiation burden. However, upon further scrutiny of this group, it became clear that a reassuring negative scan was vital in many of them, particularly those who were sedated or obtunded and those with major distracting injuries.

While CT of the head may be considered unnecessary in patients with minor traumatic brain injury, ${ }^{[16]}$ the majority of our patients in this category had risk factors necessitating 
head CT, including loss of consciousness, post-traumatic amnesia, seizure, alcohol intoxication and deformities in the craniofacial region. Regarding the need for CT of the cervical spine, physical examination of the cervical spine is unreliable in patients who are not awake and alert. ${ }^{[17]}$ Although Gonzalez et al. ${ }^{[17]}$ question the significance of distracting injuries, reputable organisations such as the Eastern Association for the Surgery of Trauma ${ }^{[18]}$ continue to caution that clinical examination is unreliable in the presence of distracting injuries. The exact definition of distracting injuries remains unclear and calls for clinical judgement. ${ }^{[19]} \mathrm{A}$ meta-analysis by Holmes and Akkinepalli ${ }^{[20]}$ showed that CT is decidedly superior to plain radiography for the diagnosis of cervical spine injuries. Pertaining to imaging of the chest, Exadaktylos et al. ${ }^{[21]}$ found multiple injuries on CT in $>50 \%$ of patients with normal initial chest radiographs, including pericardial tamponade, aortic arch injuries and lung contusions. With reference to the need for abdominal CT, physical examination of the abdomen is often unreliable in patients with associated head injury, spinal cord injury or drug ingestion, and abdominal injuries may be missed in 16 - 45\% of patients. ${ }^{[22]}$

Looking at the subgroup with clinically negative scans $(n=32$, $22.9 \%)$, there were therefore 3 patients ( $2.1 \%$ of the total) who were sedated, intubated and admitted to the intensive care unit, making further clinical assessment of the brain, spine and abdomen unreliable. Of the remaining 29 patients in this group $(20.7 \%$ of the total), 14 (10.0\% of the total) were found to have a GCS of $<15$, which rendered clinical evaluation of the cervical spine and abdomen unreliable. Of the remaining 15 patients ( $10.7 \%$ of the total), 9 (6.4\% of the total) were found to have a major distracting injury (e.g. fractured femur, fractured pelvis) that made clinical assessment of the cervical spine and possibly the abdomen suspect. In these patients, clinically negative imaging provided useful clinical information (i.e. no missed serious injuries).

This left a small subset of $4.3 \%$ of all patients who were not intubated and ventilated, were not obtunded, had no major distracting injury and in whom a combination of clinical assessment and other imaging modalities may have been sufficient to manage them without recourse to CT. Overall, 108 (77.1\%) of all scans therefore influenced initial management, a further 26 clinically negative scans $(18.6 \%$ of the total) were clinically helpful, and only 6 scans $(4.3 \%)$ were not warranted.

There were no clinically significant missed injuries in this series. However, it is important to keep in mind that pan CT does not eliminate the risk of missed injuries, and close monitoring with clinical follow-up is crucial. ${ }^{[23]}$ Defining the ideal pan CT strategy is an elusive goal; ${ }^{[24,25]}$ it may appear that pan CT in some units is often used to determine which patients can be discharged from the emergency room, while in our setting, pan CT is only performed on those who clearly need admission and for whom a management strategy needs to be formulated. Physician education may have a positive influence on a liberal attitude to the use of CT. ${ }^{[2,27]}$ Various authors suggest strategies to reduce radiation exposure in patients undergoing CT scans, ${ }^{[28-30]}$ and all units utilising CT scanning would do well to implement such measures. Using our criteria as outlined, we had a very low incidence of what could have been regarded as unnecessary pan scans.

\section{Conclusion}

As a result of this analysis, we continue to adhere to our criteria as outlined, but we consider withholding pan CT when a patient's GCS is 15 , there is no major distracting injury and no ICU admission is required.

\section{References}

1. Hutter M, Woltmann A, Hierholzer C, Gärtner C, Bühren V, Stengel D. Association between a singlepass whole-body computed tomography policy and survival after blunt major trauma: A retrospective cohort study. Scand J Trauma Resusc Emerg Med 2011;19(1):73. DOI:10.1186/1757-7241-19-73

2. Huber-Wagner S, Lefering R, Qvick LM, et al; Working Group on Polytrauma of the German Trauma Huber-Wagner S, Lefering R, Qvick LM, et al; Working Group on Polytrauma of the German Trauma
Society. Effect of whole-body CT during trauma resuscitation on survival: A retrospective, multicentre Society. Effect of whole-body CT during trauma resuscitation on survival: A retrose
study. Lancet 2009;373(9673):1455-1461. DOI:10.1016/s0140-6736(09)60232-4

study. Lancet 2009;373(9673):1455-1461. DOI:10.1016/s0140-6736(09)60232-4
Brenner DJ, Elliston CD. Estimated radiation risks potentially associated with full-body CT screening Brenner DJ, Elliston CD. Estimated radiation risks potentially asso
Radiology 2004;232(3):735-738. DOI:10.1148/radiol.2323031095

4. Brenner D, Elliston C, Hall E, Berdon WE. Estimated risks of radiation-induced fatal cancer from pediatric CT. AJR Am J Roentgenol 2001;176(2):189-196. DOI:10.2214/ajr.176.2.1760289

5. Laing GL, Skinner DL, Bruce JL, Aldous C, Oosthuizen GV, Clarke DL. Understanding the burden and outcome of trauma care drives a new trauma systems model. World J Surg 2014;38(7):1699-1706. DOI:10.1007/s00268-014-2448-8

6. Gottschalk MB, Bellaire LL, Moore T. Radiation exposure in the young level 1 trauma patient: A retrospective review. J Surg Orthop Adv 2015;24(1):57-63.

Sierink JC, Saltzherr TP, Wirtz MR, Streekstra GJ, Beenen LF, Goslings JC. Radiation exposure before and after the introduction of a dedicated total-body CT protocol in multitrauma patients. Emerg Radiol 2013;20(6):507-512. DOI:10.1007/s10140-013-1147-3

8. Tepper B, Brice JH, Hobgood CD. Evaluation of radiation exposure to pediatric trauma patients. J Tepper B, Brice JH, Hobgood CD. Evaluation of radiation exposure to
Emerg Med 2013;44(3):646-652. DOI:10.1016/.jemermed.2012.09.035

9. Kharbanda AB, Flood A, Blumberg K, Kreykes NS. Analysis of radiation exposure among pediatric

Kharbanda AB, Flood A, Blumberg K, Kreykes NS. Analysis of radiation exposure among pediatric
trauma patients at national trauma centers. J Trauma Acute Care Surg 2013;74(3):907-911. trauma patients at national traum
DOI:10.1097/ta.0b013e318287883e

10. You JS, Lee HJ, Chung YE, et al. Diagnostic radiation exposure of injury patients in the emergency department A cross-sectional large scaled study. PLoS One 2013;8(12):e84870. DOI:10.1371/journal.pone.0084870

1. Linet MS, Slovis TL, Miller DL, et al. Cancer risks associated with external radiation from diagnostic imaging procedures. CA Cancer J Clin 2012;62(2):75-100. DOI:10.3322/caac.21132

12. Sharma OP, Oswanski MF, Sidhu R, et al. Analysis of radiation exposure in trauma patients at a level I trauma center. J Emerg Med 2011;41(6):640-648. DOI:10.1016/j.jemermed.2011.03.004

3. Shah KH, Slovis BH, Runde D, Godbout B, Newman DH, Lee J. Radiation exposure among patients with the highest CT scan utilization in the emergency department. Emerg Radiol 2013;20(6):485-491. DOI:10.1007/s10140-013-1142-8

14. Jones AC, Woldemikael D, Fisher T, Hobbs GR, Prud'homme BJ, Bal GK. Repeated computed tomographic scans in transferred trauma patients: Indications, costs, and radiation exposure. J Trauma Acute Care Surg 2012;73(6):1564-1569. DOI:10.1097/ta.0b013e31826fc85f 15. Salim A, Sangthong B, Martin M, Brown C, Plurad D, Demetriades D. Whole body imaging in blunt
multisystem trauma patients without obvious signs of injury: Results of a prospective study. Arch Surg multisystem trauma patients without obvious signs of $i$

16. Sadeghian H, Motiei-Langroudi R. Does distracting pain justify performing brain computed tomography in multiple traumas with mild head injury? Emerg Radiol 2016;23(3):241-244. DOI:10.1007/s10140-016-1387-0

17. Gonzalez RP, Cummings GR, Phelan HA, Bosarge PL, Rodning CB. Clinical examination in complement with computed tomography scan: An effective method for identification of cervical spine injury. J Trauma 2009;67(6):1297-1304. DOI:10.1097/ta.0b013e3181c0b604

18. Como JJ, Diaz JJ, Dunham CM, Chiu WC, Duane TM, Capella JM. Cervical spine injuries following trauma. J Trauma 2009;67(3):651-659. DOI:10.1097/TA.0b013e3181ae583b

19. Hefferman DS, Schermer CR, Lu SW. What defines a distracting injury in cervical spine assessment? Trauma 2005;59(6):1396-1399. DOI:10.1097/01.ta.0000196006.26551.ce

20. Holmes JF, Akkinepalli R. Computed tomography versus plain radiography to screen for cervical spine injury. J Trauma 2005;58(5);902-905. DOI:10.1097/01.ta.0000162138.36519.2a

21. Exadaktylos AK, Sclabas G, Schmid SW, Schaller B, Zimmermann H. Do we really need routine computed tomographic scanning in the primary evaluation of blunt chest trauma in patients with computed tomographic scanning in the primary evaluation of blunt chest trauma in patients with
'normal' chest radiograph? I Trauma 2001:51(6):1173-1176. DOI:10.1097/00005373-200112000-00025

22. Peitzman AB, Makaroun MS, Slasky BS, et al. Prospective study of computed tomography in initial management

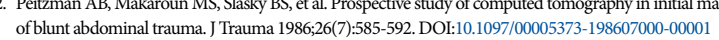

of blunt abdominal trauma.J Trauma 1986;26(7):585-592. DOI:10.1097/00005373-198607000-00001
23. Stengel D, Ottersbach C, Matthes G, et al. Accuracy of single-pass whole-body computed tomography for detection of injuries in patients with major blunt trauma. CMAJ 2012;184(8):869-876. DOI: $10.1503 / \mathrm{cmaj} .111420$

24. Gupta M, Schriger DL, Hiatt JR, et al. Selective use of computed tomography compared with routine whole body imaging in patients with blunt trauma. Ann Emerg Med 2011;58(5):407-416 DOI:10.1016/j.annemergmed.2011.06.003

25. Tillou A, Gupta M, Baraff LJ, et al. Is the use of pan-computed tomography for blunt trauma justified? A prospective evaluation. J Trauma 2009;67(4):779-787. DOI:10.1097/ta.0b013e3181b5f2eb

26. Griffey RT, Jeffe DB, Bailey T. Emergency physicians' attitudes and preferences regarding computed tomography, radiation exposure, and imaging decision support. Acad Emerg Med 2014;21(7):768-777. DOI:10.1111/acem. 12410

27. Gimbel RW, Fontelo P, Stephens MB, et al. Radiation exposure and cost influence physician medical image decision making: A randomized controlled trial. Med Care 2013;51(7):628-632. DOI:10.1097/ mlr.0b013e3182928fd

28. Sodickson A. Strategies for reducing radiation exposure from multidetector computed tomography in the acute care setting. Can Assoc Radiol J 2013;64(2):119-129. DOI:10.1016/j.carj.2013.01.002

29. Talati RK, Dunkin J, Parikh S, Moore WH. Current methods of monitoring radiation exposure from CT. J Am Coll Radiol 2013;10(9):702-707. DOI:10.1016/j.jacr.2013.03.002

30. Kalra MK, Maher MM, Toth TL, et al. Strategies for CT radiation dose optimization. Radiology 2004;230(3):619-628.

Accepted 31 May 2016. 